\title{
The Treatment of Dysentery.
}

\section{Philip Manson-Baitr.}

T $\mathrm{T}$ is by no means easy to give a concise account of the modern ideas on the treatment of dysentery without some understanding of differential diagnosis. Exact diagnosis is at the root of the whole subject, and this may be somewhat difficult.

Dysentery is best classified as a symptom-complex due to a most varied etiology.

\section{Bacillary Dysentery.}

Caused either by Shiga's bacillus, Flexner's bacillus, or, according to recent ideas, by Sonne's bacillus, has a worldwide distribution and occurs in epidemic form, as a rule. Sporadic cases do occur from time to time in Northern Europe, even in England, but it is of more frequent incidence in mental asylums and other similar institutions. In the tropics and sub-tropics and in every country with a constantly high summer temperature this disease is widespread and it is an infection to which the newcomer is especially prone.

\section{Protozoal Dysentery.}

This is caused by the invasion of the large intestine by protozoa, and of these we recognise two species, the Entamoba histolytica and the Balantidium coli, which are liable to do so. Intestinal balantidiasis is so rare that we need hardly discuss it here at all. On the other hand, amœbiasis is a very widespread disease. The term amœbiasis is used advisedly to denote the infection of the body with Entameba histolytica for this organism occasionally migrates from the bowel and becomes transplanted into the liver, lung or even brain, where it causes metastatic abscesses.

Acute cases of amœbic dysentery, that is cases with acute symptoms, diarrhœa, abdominal pain and other subjective symptoms are rare. Usually the disease assumes a chronic form, so that the average amœbic case is a much drawn out and less dramatic affair than is the bacillary disease. Once a person is infected with Entamcba histolytica unless properly treated probably remains so for life, so that cases of long-standing infection are of quite common 
occurrence. I have encountered cases who have harboured the Entamœba histolytica for 30 years or more without seriously undermining their general health.

Finally there is to be considered that form of dysentery which is due to helminthic infection. Dysenteric symptoms are especially liable to appear in persons infected with one of the bilharzia parasites in which case the eggs of the parasite in endeavouring to reach the exterior pass through the walls of the bowel producing ulceration and various forms of lesions in so doing. Naturally these much more serious infections are more troublesome to deal with.

In addition to these well-recognised more or less tropical forms of dysentery there remain a whole category of conditions, such as tuberculosis, syphilis, malignant disease and various kinds of growth of the bowel which may produce symptoms more or less indistinguishable. Treatment of these various conditions should be based upon two main principles :-

(a) Treatment directed towards the eradication of the source of the disease.

(b) Treatment directed towards the maintenance of the patient's general condition.

Bacillary dysentery, for instance, is a sharp and dramatic disease. It requires early vigorous measures, and above all, skilful nursing, not always obtainable in those parts where this type of dysentery is rife.

The dietetic question is of great importance. The patient's body is being drained of fluid by the constant diarrhœa, his tissues are being poisoned with the toxins of the causal organism. As much fluid as can be tolerated must be given by the mouth, this should consist of lemonade, barleywater, beef tea, chicken broth, meat extracts, \&c. A diet composed entirely of milk is not well tolerated.

Opium, morphia and sedatives generally should be used cautiously as a means of alleviating the distressing colicy pains, the straining or tenesmus, and in order to obtain rest and sleep, but not as a means of curing the disease. The old idea of treating bacillary dysentery by means of lead and opium pills has been relegated to the past. It was based upon putting the bowel " into splints," but, in so doing, it produces a condition of intestinal stasis and increases the absorbability of toxins from the bowel wall.

The main principles underlying treatment have been based on the opposite method. It has been found that by 
giving aperients such as salines (magnesium or sodium sulphates) the bowel is kept clean of intestinal contents, and at the same time the toxins of the bacillus which are so highly deleterious are got rid of. The generally accepted plan is to give sodium sulphate in drachm doses every two hours for the first 48 hours of the illness and thereafter four times daily till the major dysenteric symptoms have abated and until the stools have become fæculent once more. Some observers have reported equally good results with rhubarb in medicinal doses and others with castor oil. In cases where diarrhœa of a choleraic character persists after the blood and mucus stage has been passed, Colloidal Kaolin(Crooke's), or bolus alba (a mixture of charcoal and kaolin), suspended in water in doses of three teaspoonfuls every two hours has the effect of checking the flux and helps to eliminate the dysenteric toxins.

Yatren (iodine-oxyquinoline sulphonic acid) in pill form by the mouth or injected as a lavage in the strength of $2 \frac{1}{2}$ per cent. per rectum has been found to exert a beneficial effect in resistant cases.

The serum treatment of bacillary dysentery is being much more widely adopted as time goes on. The best brands of antidysenteric serum are made by the Lister Institute in this country and in America by Mulford's. Whichever brand of serum is used, the underlying principle is the same. The best sera are those which are richest in Shiga antigens, for it is generally accepted that it is these Shiga bacillus infections which are the most serious clinical cases. The serum requires to be injected in considerable doses and as early as is possible in the course of the disease. This is true when bacillary dysentery attacks small children, especially European children in whom it may lead to a rapidly fatal issue and be ushered in by convulsions.

The indications for the use of serum should be based upon the patient's general condition. If he is really ill with pyrexia, small rapid pulse, sunken painful abdomen and a malar flush it suggests that he is suffering badly from the absorption of dysenteric toxins. The frequency of the diarrhœa, i.e., more than 18 stools in the 24 hours is also important. In very severe and fulminating cases anti-dysenteric serum should be injected slowly and should be warmed and strained through sterilised filter paper first. If there is much collapse and, if the blood pressure is unduly low, a pint of normal saline should be injected as well. Precautions must be taken to guard against anaphylaxis by enquiring whether horse 
serum has been injected some time previously. Apparently the serum is absorbed more quickly when given by the intramuscular than by the subcutaneous route, and this method has the superadded advantage of being far less painful. Sometimes it may be necessary to give 50 c.c. or even more up to 100 c.c. The adductors of the thigh or the gluteus muscle may be used for the purpose. Very of ten serum treatment is complicated by the supervention, seven to ten days afterwards, of a serum rash, urticaria, accompanied by pyrexia and joint pains. It is possible to prevent the major manifestations by vigorous treatment with calcium lactate ( $20 \mathrm{gr}$. a day).

There are several complications of bacillary dysentery, the chief being dysenteric arthritis and iritis. Both are apt to supervene accompanied by pyrexia and general symptoms of intoxication during convalescence in the third week of the disease when the stools are becoming semi-formed and fæculent once again. The arthritis is best treated by general measures, such as radiant heat, massage and Scott's dressing applied to the joints; the iritis by means of an eye-shade and atropine drops. Occasionally bacillary dysentery may develop into a chronic form and this is a much dreaded complication and one which is extremely difficult to treat.

Chronic bacillary dysentery is really a chronic diarrhœa which may be accompanied by great emaciation and anæmia which develops gradually and insidiously months, it may be years, subsequent to the original attack. Many of these cases have been noted amongst war pensioners, and in whom the symptoms became manifest five, six or even more years after the Armistice, when the original infection was contracted on active service. Apart from operative measures, such as appendicostomy and cæcostomy which must always be reserved for the most intractable and otherwise hopeless cases, chronic bacillary dysentery is best treated by means of intestinal lavage. Many substances are used for irrigating the bowel, notably sodium bicarbonate and eusol. The former is given in a 2 per cent. solution in hot water and one or more pints are injected slowly by means of a tube and funnel into the rectum and permitted to penetrate the bowel. On the whole, especially when the diarrhœa is accompanied by a degree of septic absorption, eusol gives the best results. At first, the drug is given greatly diluted, one part of eusol to nine parts of water, but the strongest solution which can be tolerated is four parts eusol to six parts warm water. The injections should be given very slowly on alternate days 
and the procedure kept up till it can be ascertained by sigmoidoscopic examination that healing of the bowel is taking place. The aftercare and dietary are most important in all clinical forms of bacillary dysentery. In order to avoid post dysenteric constipation it is usually necessary for the patient to take liquid paraffin or petrolagar in teaspoonful doses at night for some considerable time. Then the diet should be carefully attended to. As a rule, the return to red meat and starchy foods, such as potatoes, cellulose containing vegetables, such as cabbages, must be gradual. Alcohol taken in the form of whisky or beer appears to have an especially deleterious effect on dysenterics and should be prohibited for at least two months after apparent complete recovery.

Frequently a post-dysenteric colitis supervenes with the passage of "unsatisfactory" stool and excess of mucus. For this condition I find the eating of Psyllium seeds (Plantago ovata) to be beneficial. The seeds are either chewed or soaked in water when a gelatinous substance exudes which acts as a demulcent. It is rather like eating canary seed, but it certainly seems to exert a most soothing effect on the bowel.

The Treatment of Amœbic Dysentery is an entirely different affair. The drug treatment is at once highly specific, and is diametrically opposed to that outlined for the bacillary disease. Hence before embarking on this highly specialised treatment it is more than ever necessary to make doubly sure that one's diagnosis is correct.

There is no doubt now to the mind of anyone that emetine (the alkaloid of ipecacuanha) has a highly selective action for the Entameba histolytica, but I am quite certain that emetine should be reserved only for the acute stage of intestinal amœbiasis and for the treatment of the major complications, such as liver abscess. Emetine is given in the form of emetine hydrochloride dissolved in ampoules of distilled water and of a capacity of 1 c.c. each. The dose for an adult of $140 \mathrm{lbs}$. $\left(63 \frac{1}{2} \mathrm{~kg}\right.$.) is one grain, and the total dosage spread over as many days for the control of the dysenteric symptoms should not exceed 10 grs. More than this, it should not be necessary to give or else toxic symptoms may supervene, and these toxic symptoms are by no means to be despised. They consist of various manifestations of which myositis and neuritis are the most severe and it must be borne in mind that some people who are specially 
susceptible to emetine are apt to develop cardiac symptoms which are sufficiently alarming.

For the more chronic forms of the disease, amœbic dysentery being essentially of a chronic and relapsing nature, the compound known as emetine-bismuthous-iodide has been introduced. This is a bright red fine powder, which when introduced into the stomach exerts powerful emetic properties. It has been used essentially in those forms of chronic amœbic dysentery in which cysts are being passed in the fæces emetine-bismuth-iodide (or E.B.I. for short) was introduced about 1916 .

As originally advised, the drug was given in gelatin covered capsules each containing 3 grains of the drug, over a course of twelve days, making a total of 36 grains altogether. As the patient was kept on a very strict dietary of milk over the whole period, the treatment was a very exhaustive one ; but in the majority of cases it succeeded in eradicating the infection where emetine injections had failed. Still relapses continued to recur with blood and mucus stools containing demonstrable entamœbæ in individuals who had been subjected to one or more courses of E.B.I. and it became customary to regard them as instances of infection with emetine-fast amœbæ. Various modifications of E.B.I. have been effected with the idea of making the drug less nauseating. The chief of these are E.P.I. (emetine periodide), and Auremetine. It is said that both these latter drugs may be given in considerably larger individual doses than E.B.I. My own experience leads me to assert that, while all are nauseating, E.P.I. is the least emetic of the three; but I am by no means convinced that it is equally as efficacious as E.B.I. The maximum dose of E.P.I. for an adult male is about 4 grains. With all these emetine compounds it is necessary to prepare the patient before the drug is given. The diet should be very light and should consist for the most part of milk (two pints), fish and chicken in the middle of the day, eggs, toast, butter and rusks. In order effectually to prevent vomiting, no solid food should be permitted for three hours before the drug is taken, and it is good practice to give a sedative such as luminal grs. 1, or Tinct. opii, minims 10, half an hour beforehand.

Since 1922, a new drug has been introduced which appears to have very powerful anti-amœbic properties. Yatren is an iodine-oxyquinolin-sulphonic acid compound. It can be given, either by the mouth or in the form of a 
small rectal enema ; from the latter situation it is absorbed and soon after is excreted in the urine where it can be recognised by appropriate tests. When given in sufficient quantities by the mouth to exert a lethal effect upon the entamœbæ, it is apt to cause a very exhausting and intractable diarrhœa and on this account its administration has been abandoned, except as a form of after-treatment. In this manner, I find that it is very useful when given in pill form (four grains each), for one month subsequent to more active measures. In my own opinion, in order to obtain a permanent cure (for that is what one is aiming at) yatren should bo given by the rectum in the form of rectal injections. I have now carried this out in over 200 cases with eminently satisfactory results. The lumen of the bowel must first be cleared of mucus and intestinal contents by means of an enema (one pint) of 2 per cent. sodium bicarbonate and 20 minutes to half an hour later the Yatren solution is injected. This is made by dissolving five grammes of Yatren in eight ounces of warm water, which results in a sherrycoloured solution. This is injected slowly by means of a catheter and funnel. The Yatren solution should be retained in the bowel as long as is possible, a period usually of six to eight hours during which it will percolate through the large intestine, coming into contact with amœbic lesions which it instantly heals up (as can be observed by frequent sigmoidoscopic examination). When excreted once more the Yatren solution appears as a greenish, slimy fluid, due probably to liberation of iodine. Yatren is practically non-toxic and the only disagreeable effect so far observed has been a flannely or roseolar rash which is apt to appear on the day following treatment. As there is no absolute evidence that Yatren solution invariably heals all the amœbic lesions in the large bowel, especially when it does not come into contact with them in the upper portion, I have been, during the last six years, combining both the Yatren and E.B.I. treatments, giving a total of 27 grains E.B.I. and ten enemeta of Yatren on as many successive days. The end results of this combined treatment, have been eminently successful, so that I have records only of two subsequent parasitic relapses, both of whom have been permanently cured by a further Yatren course. In these especially intractable cases the Yatren solution may be used as strong as 5 per cent. without giving rise to any inconvenience.

As an after cure of intestinal amœbiasis, I use Stovarsol in small doses. This is an arsenical preparation (Acetyl-oxy-amino-phenyl-arsenic acid), it has acquired 
a considerable reputation as a cure for amœbic dysentery in France, but there is no actual proof that it exerts any direct action upon the amœbæ themselves. Probably it is a very distinct intestinal tonic ; it braces up the system and makes the patient feel better. I usually give it in one tablet a day for 14 days subsequent to active treatment, but I consider it very necessary that care should be exercised in prescribing Stovarsol. In some people it is very liable to produce toxic symptoms and curious rubeolar arsenical rashes are comparatively common after its use. Therefore, the wise man should not prescribe more than one tablet a day. This treatment is best reinforced by Yatren of which one pill should be given at night for one month. As regards diet, in the after treatment of intestinal amœbiasis, I consider that it is necessary to exert a moderate amount of care for one month or so. One red meat meal, as beef or mutton, may be allowed for lunch, whilst chicken, fish and eggs, milk, milk puddings and green vegetables should make up the major portion of the dietary. It is a sensible measure to interdite all alcohol for one month.

Patients who have suffered from amœbic dysentery are apt to be distressed with flatulency for some time af terwards ; they may have a tendency to constipation and be forced to take petrolagar or liquid paraffin at night, but in my experience when once vigorously treated, the bowel action soon becomes restored to normal and the patient is not plagued by stenosis, adhesions and all kinds of malformations such as are described in the older textbooks of medicine.

Finally, regarding the third class of dysentery-the helminthic dysenteries - where these are due to intestinal bilharziasis, it is necessary to extirpate the parent bilharzia worms themselves as they are the source of the trouble. This is done by intravenous injections of tartar emetic, commencing with small doses of half-grain each, and gradually working up to two-and-a-half grains. A total course of treatment of 30 to 40 grains appears to be necessary in the case of intestinal bilharziasis; but intravenous therapy alone does not, of course, entirely cure the intestinal lesions, for where there are large polypi, it may be necessary to have recourse to surgical measures in order to remove them.

As I remarked at the commencement of this paper, dysentery is a very large subject, and if I were to attempt to describe those conditions which simulate true dysentery closely and to embark on a discussion of their treatments, I am afraid I should overstep all bounds. 